\title{
Linx
}

Revue des linguistes de l'université Paris X Nanterre

5 | 1994

La négation

\section{La négation et l'hypothèse inaccusative}

\section{Francisco Hernández-Paricio}

\section{(2) OpenEdition}

\section{Journals}

Édition électronique

URL : http://journals.openedition.org/linx/1197

DOI : 10.4000/linx.1197

ISSN : 2118-9692

\section{Éditeur}

Presses universitaires de Paris Nanterre

\section{Édition imprimée}

Date de publication : 1 juin 1994

Pagination : $91-120$

ISSN : 0246-8743

\section{Référence électronique}

Francisco Hernández-Paricio, «La négation et l'hypothèse inaccusative », Linx [En ligne], 5 | 1994, mis en ligne le 18 juillet 2012, consulté le 30 avril 2019. URL : http://journals.openedition.org/linx/1197 DOI : 10.4000/linx.1197

Ce document a été généré automatiquement le 30 avril 2019.

Département de Sciences du langage, Université Paris Ouest 


\title{
La négation et l'hypothèse inaccusative
}

\author{
Francisco Hernández-Paricio
}

\section{L'hypothèse inaccusative}

1 Avec quelques différences d'ordre terminologique, l'hypothèse inaccusative, proposée à l'origine dans le cadre de la Grammaire Relationnelle (Perlmutter, 1978) a été adoptée plus tard par les tenants du modèle de GB [Rection et Liage], et très particulièrement après les remarques de Burzio (1981 et 1986), Belleti et Rizzi (1982) et Belletti (1988).Étant donné le but de notre travail, c'est la version de l'hypothèse présentée par Belletti (1988) qui sera notre point de repère, de façon que les aspects que nous allons aborder ont comme point de départ direct les conclusions qui sont exposées dans cette étude ${ }^{1}$.

2 L'Hypothèse Inaccusative établissait fondamentalement une division, à l'intérieur du groupe des prédicats intransitifs, entre deux classes: celle des intransitifs dont l'argument est considéré sujet dans le niveau syntaxique profond (les prédicats « inergatifs ») et celle des prédicats dont l'argument montre plutôt le comportement d'un objet direct (les «inaccusatifs» ou "ergatifs» de Burzio). Je maintiendrai le terme « inaccusatif », pour éviter de confondre les différents signifiés du terme " ergatif ».

3 L'exemplification des différences entre ces deux types de prédicats peuvent être résumées dans l'argument de Burzio, qui dit que, dans une langue à inversion libre du sujet (comme l'italien et l'espagnol, qui appartiennent au paramètre du sujet nul), on peut trouver des SN de nature différente postposés au V : certains qui apparaitraient dans cette position postverbale à cause d'un véritable mouvement de postposition (adjoints au V), tandis que d'autres occuperaient cette position parce qu'ils y auraient été directement générés. Ce sont ces derniers $\mathrm{SN}$ qui correspondent à des inaccusatifs : des prédicats à un argument, réalisé par un SN directement généré en position d'objet (c'est à dire postverbale), et pour lequel la position préverbale serait « dérivé ».

4 Pour prouver que les SN postposés arguments d'inaccusatifs présentent l'aspect des SN objets on a recours à la preuve typique de la possibilité d'extraction à l'aide du clitique en 
(possibilité restreinte aux SN postposés, en fonction d'objets de V transitifs), comme on peut constater dans les exemples de (1) y (2)

\begin{tabular}{|l|l|}
\hline (1) & a. Arriveranno molti esperti \\
\hline & b. Ne arriverano molti \\
\hline (2) & a. Telefoneranno molti esperti \\
\hline & b. ${ }^{*}$ Ne telefoneranno molti \\
\hline
\end{tabular}

5 Une autre des caractéristiques le plus utilisée dans la bibliographie est l'association des $\mathrm{V}$ inaccusatifs avec l'auxiliaire essere, face aux inergatifs qui s'associent à avere [ou les formes correspondantes dans les langues qui possèdent une distinction parallèle :

(3) Sonno arrivati molti esperti

(4) Hanno telefonato molti esperti.

6 Pour les langues qui n'appartiennent pas au paramètre du sujet nul, on insiste sur la possibilité d'obtenir des constructions impersonnelles du genre de (5) :

(5) Il est venu trois filles.

7 avec un explétif dans la position du sujet (comme l'anglais it ou there), ou d'autres genres de procédés dont le résultat est l'inversion du sujet, c'est-à-dire, sa construction en position postverbale.

8 Dans ce sens, l'espagnol n'est pas tout à fait semblable à l'italien, puisque, malgré l'appartenance des deux langues au paramètre du sujet nul, l'espagnol manque de formes semblables au ne it. ou catalan et le fr. en (formes clitiques utilisées dans les extractions de compléments de quantificateurs dans des SN objets ou sujets en position postverbale). L'espagnol ne compte qu'un seul auxiliaire pour tous les verbes (haber), et bien entendu il n'a pas la possibilité de formations impersonnelles du type de (5). Cela ne veut pas dire que l'inaccusativité n'ait pas lieu en espagnol, mais seulement que son identification doit faire appel à d'autres recours.

\subsection{Le cas des sujets-objets des inaccusatifs}

9 La situation des SN arguments de V inaccusatifs, à part la reconnaissance de leur statut d'objets profonds et donc sujets "dérivés", était celle d'un argument externe quelconque. Dans ce sens, les arguments de Burzio, ou ceux de May (1985) par exemple, ne visaient qu'à justifier l'assignation du cas nominatif (en général considéré comme structural) à un argument qui, en tant qu'objet profond, avait la condition d'argument interne. 
10 Le recours habituel, dans ce cas, était une extension de l'analyse proposée pour les langues présentant une forme explétive dans le sujet. Recours qui justifie l'existence d'une chaîne dont le premier élément serait l'explétif ou une catégorie vide (suivant le paramètre auquel appartient la langue) en position externe qui transmettrait le cas nominatif structurel au second élément de la chaîne, c'est à dire, au SN, avec des structures comme celle de (6), de Burzio

(6) $\mathrm{P}[\mathrm{SN}[\mathrm{e}] \mathrm{SV}[$ arriva $] \mathrm{SN}[$ Giovanni $]]]$

D’après les analyses de Belletti et Rizzi (1982), May (1985:66) proposait pour des phrases comme (7) et (8) les structures de (9) et (10), respectivement :

\begin{tabular}{|l|l|}
\hline$(7)$ & *Ne hanno parlato tre \\
\hline$(8)$ & Ne sonno arrivato tre \\
\hline$(9)$ & {$\left[e\left[\mathrm{VP}\left[\mathrm{VP} \mathrm{ne}_{i}+\mathrm{V} \ldots\right]\left[\mathrm{NP} \mathrm{Q} e_{i}\right]\right]\right]$} \\
\hline$(10)$ & {$\left[e\left[\mathrm{VP} \mathrm{ne}_{i}+\mathrm{V} \ldots\left[\mathrm{NP} \mathrm{Q} \mathrm{e}_{i}\right]\right]\right]$} \\
\hline
\end{tabular}

Dans (7) ne est uni à V, il ne c-commande pas sa trace, qui, elle, est contenue dans le SN adjoint au SV, et c'est donc le liage propre qui fait défaut. Dans (8), au contraire, $\boldsymbol{n} \boldsymbol{e}$ ccommande sa trace, qui est dans une position structurellement non-distincte de celle d'un objet. Il s'agit donc d'une structure qui met à l'évidence que la relation manifestée par ne est identique a celle qu'il manifesterait lorsqu'il y a eu extraction d'un objet. La catégorie vide initiale serait responsable de ce que le SN postposé réalise le Cas nominatif.

\subsection{Les arguments de Belletti (1988)}

13 Tout en tenant compte de la classification de V inaccusatifs, Belletti (1988) ébauche des arguments contraires à l'assignation généralisée du cas nominatif. Les différences fondamentales ont trait aux aspects suivants :

- Il existe un certain type de rapport entre le Definiteness Effect ${ }^{2}$ et les caractéristiques des prédicats inaccusatifs.

- Les prédicats inaccusatifs peuvent assigner un cas inhérent aux SN générés en position d'objet.

- Le cas inhérent que ces prédicats assignent n'est pas le nominatif, mais le partitif.

- Nous allons considérer très brièvement chacun de ces aspects.

\subsubsection{L'effet indéfini (Le Definiteness Effect)}

Milsark (1974 et 1977), à propos des phrases existentielles, établit le point de départ de ce qui plus tard a été connu comme le Definiteness Effect (l'effet indéfini [EI]). Cet effet est observable à partir des restrictions présentées par certaines constructions, du fait qu'elles n'admettent que des objets indéfinis. Pour ce qui est des phrases existentielles, on peut penser à des exemples comme ceux de (11) : 


\begin{tabular}{|l|l|}
\hline$(11)$ & a. There is a man in the room \\
\hline \hline & b. Il y a un homme à la porte \\
\hline & c. Hay un hombre en el ascensor \\
\hline & d. Hi ha un home a l'aparell \\
\hline & e. C'è un uomo in ascensore \\
\hline
\end{tabular}

Tous ces exemples montrent l'effet indéfini, puisqu'ils ne pourraient pas être construits avec des SN définis ${ }^{3}$ :

\begin{tabular}{|l|l|}
\hline (12) & a. ${ }^{*}$ There is the man in the room \\
\hline & b. ${ }^{*}$ Il y a l'homme à la porte \\
\hline & c. ${ }^{*}$ Hay el hombre en el ascensor \\
\hline & \\
\hline & d. Hi ha l'home a l'aparell [non existentielle] \\
\hline & e.*'C'è l'uomo in ascensore \\
\hline
\end{tabular}

Il ne s'agit pas d'une impossibilité absolue, dans tous les cas, de combinaison avec des SN définis ${ }^{4}$, puisqu'il faudrait tenir compte d'autres types de spécification qui pourrait être accomplie par d'autres éléments non-déterminants ${ }^{5}$, ainsi que du fait que l'expression de cette "existentialité" peut très bien faire appel à d'autres mécanismes dans les différentes langues. Ce dont il faut tenir compte, c'est la fréquence avec laquelle ces types de phrases, à mi chemin entre existentielles et présentationnelles, s'associent à des SN dont la référence est non spécifique.

D'après Belletti, ces types de phrases, de même que ce que Milsark appelait «inside verbals » (comme arise ou develop),

\begin{tabular}{|l|l|}
\hline$(13)$ & There arise $\left({ }^{*}\right.$ the $)$ typhoons here. \\
\hline$(14)$ & a. There developed a $\left(/ *^{*}\right.$ the $)$ serious problem. \\
\hline & b. Se planteó un $\left(/{ }^{*} \mathrm{el}\right)$ serio problema \\
\hline
\end{tabular}

ont le même aspect que les verbes que Burzio considérait comme "ergatifs", et ils semblent avoir des comportements tout à fait semblables. C'est pour cela que Belletti considère de la même façon les exemples de verbes inaccusatifs traditionnels et les phrases existentielles (ainsi que le verbe qui y apparaît), étant donné que dans les deux cas il est possible de trouver des SN non définis : 
\begin{tabular}{|l|l|}
\hline (15) & a. Estuvieron llegando artistas hasta las tantas de la noche. \\
\hline \hline & b. Hay hombres armados por todas partes. \\
\hline
\end{tabular}

19 Il est évident que l'élargissement du concept d'inaccusativité entraîne l'abandon de l'exigence de l'interprétation indéfinie pour les SN concernés, mais ceci n'a pas une très grande importance dans l'argumentation de Belletti. Il est même possible que cet abandon devienne inévitable, si on veut rendre compte d'une plus grande quantité de faits dans un plus grand nombre de langues.

20 Même si la fréquence de l'association entre $\mathrm{V}$ inaccusatifs et interprétation indéfinie des SN arguments est généralement acceptée, on ne trouve pas souvent des réflexions portant sur les caractéristiques de ces prédicats qui pourraient rendre favorable ou exiger, selon le cas, l'effet indéfini. Dans le cas des phrases existentielles, il semble raisonnable d'envisager que la mention de l'existence d'une entité puisse être interprétée comme une mention initiale et introductrice d'un élément qui n'a pas "encore » été défini [ce que Givon appellerait « indéfini important »], et la même argumentation serait valable pour les présentationnelles ou les structures qui font intervenir un processus quelconque produisant le même type de "présentation ». Les inaccusatifs ou ergatifs de Burzio, avec des paramètres assez variables parmi les différentes langues, semblent plutôt difficiles à systématiser de façon unitaire. Pour ce type de prédicats, l'interprétation indéfinie reste seulement une possibilité, même si elle est utilisée par Belletti comme caractéristique essentielle ${ }^{6}$. Cependant, nous pouvons déjà avancer que le rapprochement entre les constructions existentielles et le reste des inaccusatifs est limité aux seuls cas où ces derniers s'associent à des $\mathrm{SN}$ indéfinis.

\subsubsection{L'assignation de cas}

21 Les circonstances relatives à l'assignation de cas dont nous avons parlé auparavant subsistent. C'est à dire qu'il est toujours nécessaire de déterminer quelle est la situation d'un SN indéfini qui présente en surface les caractéristiques d'un objet et qui est, de fait, traité comme un objet profond.

En tenant compte du fait que les prédicats inaccusatifs ont un seul argument, le SN qui doit le réaliser devra être marqué au moyen d'un certain cas. Si on propose une assignation de cas limité à des langues à sujet obligatoire (avec des explétifs en position de sujet, donc), les arguments de Burzio continueraient à être valables, et le SN indéfini pourrait recevoir le cas nominatif de l'élément initial de la chaîne.

$23 \mathrm{Si}$, au contraire, on prétend renforcer l'idée que ces SN indéfinis sont en effet générés en position d'objet et qu'aucun explétif ni catégorie vide en position de marque de cas structural ne lui assigne de cas, il faut, comme Belletti le fait, préconiser que, de même que cela peut arriver avec les compléments, ces SN ont un cas assigné de façon inhérente par le prédicat.

24 Il faut remarquer que si l'on considère les SN dont on parle comme des objets, cela suppose une incapacité à recevoir un cas structurel. Si ces SN n'étaient pas vraiment des objets internes du SV, la discussion n'aurait pas de sens. Par conséquent, il faut préciser à nouveau que, en dehors des arguments de Belletti, les faits syntaxiques prouvent que les 
SN définis peuvent (avec des verbes inaccusatifs, et dans une langue où l'inversion du sujet est possible) apparaître en position préverbale et postverbale :

\begin{tabular}{|l|l|}
\hline (16) & a. Afortunadamente, llegaron las cartas a tiempo. \\
\hline & b. Afortunadamente, las cartas llegaron a tiempo. \\
\hline
\end{tabular}

25 Ces mêmes langues ne permettent pas, en général, l'antéposition, dans la position habituelle du sujet, d'un SN indéfini :

\begin{tabular}{|l|l|}
\hline$(17)$ & a. Llegaron cartas y telegramas. \\
\hline & b. *Cartas y telegramas llegaron. \\
\hline
\end{tabular}

Dans des langues à sujet obligatoire, il est possible de construire des phrases impersonnelles à sujet explétif et des SN indéfinis en position interne au SV, mais elles ne permettent pas des SN définis dans cette position :

\begin{tabular}{|l|l|}
\hline$(18)$ & a. Il est arrivé trois filles. \\
\hline \hline & b. * Il est arrivé Marie \\
\hline
\end{tabular}

27 Il serait donc, d'un certain point de vue, naturel de considérer que les SN indéfinis postposés sont de véritables objets. Et pour des langues à sujet nul, il n'est pas obligatoire de supposer qu'ils reçoivent le cas nominatif, puisqu'on peut même signaler des exemples de refus. Par exemple, le catalan, qui admet des SN définis dans des phrases de caractéristiques semblables à celles des existentielles (voir note 4), refuse l'apparition de pronoms nominatifs (exemples de Rigau (1988)) :

\begin{tabular}{|l|l|}
\hline (19) & a. Hi ha un noi \\
\hline \hline & b. Hi ha el noi \\
\hline \hline & c. Hi ha en Pere \\
\hline & d. ${ }^{\text {Hi ha ell }}$ \\
\hline
\end{tabular}

Il existe des exemples de constructions présentationnelles qui ont tendance à exclure le cas nominatif (sujet) :

\begin{tabular}{|l|l|l|}
\hline$(20)$ & a. & C'est moi \\
\hline & b. & It's me \\
\hline
\end{tabular}


quoiqu'il soit vrai qu'il y a d'autres langues (suivant le paramètre du sujet nul) où le pronom semble présenter le cas nominatif :

\begin{tabular}{|l|l|l|}
\hline$(21)$ & a. & Soy yo \\
\hline & b. & Sono io. \\
\hline
\end{tabular}

ou des constructions à l'accusatif, comme celles de l'espagnol haber. Bien que ce qui semble être vraiment important soit, dans tous les cas, le fait de trouver des SN ou des pronoms postposés au verbe.

\subsubsection{Le cas partitif}

La différence de comportement des SN indéfinis, c'est à dire leur condition d'objets, et la ressemblance que Belletti croit trouver entre ces cas et les expressions existentielles, lui permet de postuler que les $\mathrm{V}$ inaccusatifs (et la forme copulative dans le cas des existentielles) ont la capacité d'assigner le cas inhérent partitif ${ }^{8}$. En fait, dans le travail de Belletti, il est supposé qu'il y a correspondance entre «SN qui reçoit le cas inhérent partitif » et "SN indéfini », ce qui est tout à fait normal dans une conception qui prétend qu'il faut attribuer la lecture indéfinie de ces $\mathrm{SN}$ au cas partitif qui leur est assigné par le $\mathrm{V}$ inaccusatif et qui donne lieu à une signification proche de celle qu'on obtiendrait à l'aide du quantificateur quelque (esp. alguno, angl. some) ${ }^{9}$.

La seule position dans un SV à verbe inaccusatif où le cas partitif puisse être assigné est celle d'objet, et l'EI ne saurait être trouvé que dans des SN qui occupent cette position. C'est le cas, par exemple, des constructions françaises avec il impersonnel, qui sont possibles avec des verbes inaccusatifs (voir (18)), avec l'EI que l'on connait, mais qu'on ne peut pas obtenir à partir de verbes inergatifs :

(22) *Il a parlé trois filles

31 De même dans des constructions passives-impersonnelles comme celles de

\begin{tabular}{|l|l|}
\hline$(23)$ & a. Il a été tué un homme \\
\hline \hline & b. Il a été tué trois candidats \\
\hline
\end{tabular}

32 Dans ce dernier cas nous trouvons des verbes qui ne peuvent pas être qualifiés d'inaccusatifs, mais qui, néanmoins, manifestent aussi l'EI. Ceci est dû au fait que la morphologie passive (malgré ses effets sur la capacité d'un verbe d'assigner le cas accusatif), n'affecte pas la capacité d'assigner un cas inhérent. C'est le verbe, donc, qui doit «choisir » le cas nominatif/accusatif ou partitif en fonction de la lecture (défini ou indéfinie) qu'on sélectionne pour le $\mathrm{SN}^{10}$.

On peut constater qu'il s'est produit un changement substantiel dans ce point, puisque l'EI a la possibilité d'être assigné par un verbe non inaccusatif. Il n'est pas moins évident que les exemples de (23) montrent des cas de morphologie passive (qui pourrait 
parfaitement être liée à des cas nominatifs), mais surtout des cas de construction impersonnelle. Et ce type spécial de construction est la raison de ce que l'on trouve des $\mathrm{SN}$ en position d'objet, car l'explétif $i$ remplit la position de sujet.

Et ce n'est pas le seul cas. Belletti même signale qu'un SN nu (pluriel) peut manifester le cas partitif, comme c'est évident si on remarque la possibilité de lui substituer le clitique $\boldsymbol{n} \boldsymbol{e}$, et l'impossibilité parallèle d'utiliser les clitiques accusatifs :

\begin{tabular}{|l|l|}
\hline$(24)$ & a. Oggi non ho scritto lettere \\
\hline & b. Lettere, oggi non ne ho scritte \\
\hline & c. ${ }^{*}$ Lettere, oggi no le ho scritte \\
\hline
\end{tabular}

En espagnol, langue qui manque de clitiques « partitifs », on remarque le même effet :

\begin{tabular}{|l|l|}
\hline$(25)$ & a. Hoy no he escrito cartas \\
\hline & b. Cartas, hoy no (*las) he escrito \\
\hline$(26)$ & a. Decisiones, hoy no (*las) hemos tomado. \\
\hline & b. Decisiones, hoy no se han tomado muchas. \\
\hline
\end{tabular}

36 Ces cas, entre autres, d'EI dans des objets de prédicats non inaccusatifs nous poussent à penser qu'il faut séparer les faits en rapport avec la définitude des SN des faits en rapport à l'inaccusativité, ou alors spécifier plus clairement le contexte où nous nous trouvons à chaque instant, et considérer davantage les raisons de l'apparition du EI. Il serait peutêtre temps de résumer et d'énoncer des divergences.

D'une part, on a une interprétation non définie (EI) de certains syntagmes. On a aussi un ensemble de situations ou de constructions favorables à cette même interprétation. Ces situations se bornent à la position d'objet dans certaines constructions qu'on peut énumérer, d'après ce qui a été vu jusqu'ici :

\begin{tabular}{|c|l|}
\hline a & Constructions existentielles \\
\hline b & Constructions impersonnelles et passives- impersonnelles \\
\hline \hline c & Constructions à V inaccusatif \\
\hline d & Constructions à V transitif \\
\hline
\end{tabular}

Il y a une différence évidente dans le fait que, dans les deux derniers cas, le EI des SN objets n'est pas obligatoire. Mais, en fait, ce ne sont pas les différences qui nous intéressent, mais plutôt la possibilité de vérifier quelles sont les ressemblances qui permettent de mettre en rapport, d'une façon raisonnable, ces différentes structures. 
39 Et il n'y a qu'une seule et simple réponse : dans tous les cas, il s'agit de constructions qui ont un verbe et un objet qui admet ou exige l'EI. Ce qu'ils ont en commun donc, c'est la présence de l'objet, ou, plus exactement, d'un SN en position postverbale. Et ce qu'il y a de remarquable dans les trois premiers cas (existentielles, impersonnelles et inaccusatifs) est que le dit SN correspond, en fait, à ce qu'on a l'habitude de considérer comme des « sujets réels ", ou même à de vrais sujets accordés dans certaines langues.

Dans ce sens, on pourrait accepter une interprétation unique pour les trois types de constructions qui ont, sans aucun doute, des ressemblances.

41 Par contre, l'apparition d'une interprétation non spécifique pour les SN concernés, ne différencie pas suffisamment les $\mathrm{V}$ inaccusatifs de tous les transitifs, en ce que cette interprétation n'est qu'une des possibilités. Il s'ensuit que la thèse qui propose l'assignation de cas inhérent partitif aux SN indéfinis n'est pas tout à fait sans problème.

42 On pourrait déjà tirer quelques conclusions provisoires :

1) Tout d'abord, on peut assurer que l'EI, vu comme l'exigence d'une interprétation indéfinie de certains objets, n'est pas obligatoirement lié au caractère inaccusatif d'un prédicat, puisque ses arguments pourraient être autant indéfinis que définis.

2) Il existe, néanmoins, un EI, vu comme l'exigence de ce que les SN indéfinis dépendants de $\mathrm{V}$ inaccusatifs occupent la position d'objet, ou plus exactement, qu'ils apparaissent en position postverbale en surface.

3) En fait, cette dernière interprétation de l'EI est parfaitement compatible (et peut-être elle y trouve son explication) avec la tendance observée dans les langues à interdire la position de sujet préverbal aux SN indéfinis.

L'EI (au sens restreint que nous venons de lui donner) s'attache fondamentalement à ce qu'on pourrait appeler les « sujets préverbaux impossibles ", c'est-à-dire à des SSNN qui ne peuvent pas occuper cette position préverbale a cause de leur caractère indéfini. Ce qui prouve que l'on doit tenir compte même des $\mathrm{V}$ transitifs étant donné que la transformation passive entraîne le déplacement de SSNN qui seraient soumis aux mêmes restrictions (quand ils doivent accomplir la fonction de sujet). Les différents moyens que les langues peuvent présenter pour s'adapter à ces restrictions sont essentiellement liés à leur appartenance à différents paramètres du sujet. Autrement dit, les langues à sujet nul, qui permettent l'inversion du sujet, auront des moyens différents de ceux des langues à sujet obligatoire pour atteindre un but qui est le même: l'apparition en position postverbale des SN indéfinis.

44 Le recours à l'assignation inhérente d'un cas partitif présente à l'avance une difficulté majeure : les $\mathrm{V}$ doivent être capables de différencier des lectures définies ou indéfinies des SN. Et, surtout, cela suppose, à mon avis, un énoncé de GU [Grammaire Universelle] qui n'a pas été suffisamment confronté avec les faits, et qui doit être mis en rapport plus étroit avec le paramètre du sujet nul.

45 En somme, il devrait être évident que les phénomènes en rapport avec l'interprétation indéfinie des $\mathrm{SN}$ ne concerne pas seulement le contextes inaccusatifs. Ils relèvent du problème général de la définitude et de la référentialité et subissent l'influence du caractère impersonnel des constructions que nous avons vu. Le fait qu'elles servent à introduire ou présenter, comme diverses questions d'ordre et de portée dépassent sans aucun doute les limites étroites de l'inaccusativité.

46 L'une des réponses possibles aux problèmes liés à l'EI (au delà les types de prédicats) repose sur le fait que l'interprétation indéfinie d'un SN est due, de façon caractéristique, a 
son utilisation habituelle pour faire référence à des objets introduits dans la situation et dans le discours pour la première fois. Des objets dont l'existence n'était pas présupposée, et qu'on interprète comme des objets « nouveaux $»^{11}$, ou des objets auxquels on fait une allusion volontairement non spécifique. Cette indéfinitude et cette non-spécificité ont pour conséquence que les SN qui les reflètent ne soient pas perçus comme des « participants » à plein droit, qu'ils apparaissent non comme objets d'une prédication, mais comme des prédications, et que, du point de vue syntaxique, ils présentent plus souvent l'aspect d'un cas oblique que celui d'un nominatif ou accusatif. Une telle situation nous invite à nommer ce cas justement « inaccusatif ».

\section{Inaccusativité et négation}

Mettre en rapport l'étude de la négation et celle des faits dont nous parlons peut avoir deux buts distincts :

a) D’une part, vérifier si en considérant l'intervention de la négation verbale dans la phrase, on constate des résultats qui pourraient renforcer ou invalider certains aspects de l'hypothèse inaccusative.

b) Et, d'autre part, établir les conditions d'intervention de cette négation verbale et leur répercussion sur la thèse qui soutient la régularité de traitement des SN indéfinis dépendants de certains types de prédicats.

En réalité, les phénomènes qu'on a commentés et la négation ont déjà été mis en rapport, même si c'était fait de façon inconsciente, dans de nombreux travaux consacrés à des aspects de la négation. Cela dit, un travail m'a beaucoup frappé, surtout parce qu'il est paru longtemps après que j'ai commencé cette étude : c'est l'article de Gaatone (1992) où il étudie ce qu'il considère comme le déterminant $d e$. Dans cet article il nous offre un parfait synopsis des phénomènes abordés jusqu'ici.

Comme Gaatone le signale, la distribution de de obéit à la contrainte bien connue : il ne peut précéder qu'un objet direct en contexte de négation verbale. Mais ce de négatif peut intervenir dans d'autres contextes. Il peut, par exemple, introduire les « sujets réels » des constructions impersonnelles, et d'autres types de constructions qui, par des moyens différents, produisent des résultats pareils (toujours sous l'effet de la négation) :

\begin{tabular}{|l|l|}
\hline$(27)$ & il ne vient plus de touristes \\
\hline$(28)$ & il n'a pas été donné de réponse \\
\hline$(29)$ & il ne faut pas d'argent ; \\
\hline$(30)$ & il n'y a jamais de visiteurs ici. \\
\hline$(31)$ & $\begin{array}{l}\text { Jamais ne se présentera d'occasion aussi décisive de démontrer l'efficacité économique... } \\
\text { Express intern., 1627. }\end{array}$ \\
\hline$(32)$ & $\begin{array}{l}\text { On n'a pas fait venir de médecin. On ne laisse pas entrer de mineurs. Je n'ai pas entendu } \\
\text { d'oiseaux chanter }{ }^{12}\end{array}$ \\
\hline
\end{tabular}



avec ce que nous avons commenté jusqu'ici: il s'agit de verbes inaccusatifs, de constructions impersonnelles obtenues à partir de verbes de ce type, ou de passifs impersonnels, et d'existentielles. Il serait relativement compréhensible de se laisser entraîner à penser que le "partitif » français correspond, grosso modo, au partitif apparemment assigné par les verbes inaccusatifs. Mais, à vrai dire, les propos de Gaatone sont encore plus intéressants, puisqu'il regroupe tout naturellement les cas qu'il présente autour de l'expression de l'impersonnalité, et il ramène le phénomène à une caractéristique commune à tous les cas, c'est à dire, qu'ils « ont en commun de mettre en jeu des arguments directs du verbe, simple ou complexe, dans une position postverbale. » (p. 96). En contexte de négation verbale, bien entendu.

51 En effet, le fait que la position des SN qui pour Belletti manifestaient l'EI soit justement postverbale est la cause directe de ce qu'ils deviennent un objet d'étude privilégié dans les recherches sur la négation, puisque, dans une telle position, ces SN se trouvent sous la portée de la négation verbale, d'autant plus qu'ils ont un caractère indéfini.

L'étude déjà citée de Perlmutter, et l'étude précédente de Gaatone (1970) établissaient clairement que ce qui est prioritaire est l'ordre des constituants en surface. Ordre qui détermine les différentes possibilités permises par les traits de définitude des SN, et qui est déterminant pour l'analyse des phénomènes de portée. Ceci fait qu'un $\mathrm{SN}$ en position postverbale présente des caractéristiques semblables à celles d'autres $\mathrm{SN}$ dans cette même position, indépendamment de leur fonction et des procédés par lesquels ils sont parvenus à occuper cette position. Et en ce qui concerne la négation, la fonction de SN n'a aucune importance : s'il est indéfini il peut être atteint par la négation, autant comme objet que comme sujet. La véritable restriction concerne le manque de liberté d'un SN indéfini pour occuper des positions préverbales, et ceci pousse à penser que l'interprétation adéquate de cette indéfinitude dépend, de façon décisive, de ce qu'il occupe une position telle qu'il puisse être contrôlé par un quantificateur. Cette fonction de quantification pourrait très bien être accomplie par la négation et/ou certains des contenus de la forme verbale, que ce soit le temps ou l'aspect, par exemple.

\subsection{Les cas du russe et du basque}

D'autre part, le rapport entre interprétation indéfinie d'un SN et négation, ne se limite pas à des questions d'ordre et de portée qui ne sont pas toujours directement discernables, mais il peut présenter des reflets formels qui le rendent évident. Pour exemplifier ce dernier cas, j'introduirai des remarques extraites de l'examen de deux langues essentiellement différentes en plusieurs sens, mais surtout dans un sens que je considère comme très significatif : il s'agit d'une langue sans déterminants (le russe) et d'une autre dont l'une des caractéristiques principales est de posséder un système établi de déterminants (le basque). Les deux langues présentent aussi des réalisations de cas morphologiques. J'utiliserai la terminologie habituelle pour chacune des langues.

\subsection{Le génitif russe}

D’après les descriptions les plus habituelles, le russe présente la particularité d'associer le cas génitif aux objets de verbes transitifs sous l'influence de la négation. Mais on ne peut pas généraliser cette association avec autant de simplicité ${ }^{13}$. Heldner (1992), par exemple, 
rappelle la distinction établie par Babby (1978) pour le russe entre des phrases existentielles et déclaratives.

2.2.1. D'après Babby, les existentielles affirmatives assertent l'existence du référent du SN sujet. Si elles sont négatives, elles assertent que le sujet ne possède pas de référent identifiable. C'est à dire que les SN qui apparaissent dans de phrases existentielles rentrent dans le champ de l'assertion. Et si on trouvait une phrase transitive à emploi existentiel, ce serait les objets qui se trouveraient dans le champ de l'assertion, mais non pas les sujets.

Dans les déclaratives, par contre, on trouve des SN (sujets ou objets) qui impliquent l'existence de leurs référents. Si la phrase est négative, on asserte la non- réalisation de ce qui est dénoté par le verbe, ce qui fait que les SN se retrouvent hors du champ de l'assertion et aussi de la négation.

Une telle différentiation a pour objet d'expliquer l'emploi du génitif pour les positions d'objet ou sujet dans les phrases existentielles négatives, alors que dans les déclaratives on aurait le nominatif pour le sujet, et l'accusatif pour l'objet.

À vrai dire, la situation schématisée par Babby (comme Heldner, elle même, le signale) est loin d'être claire, en ce qu'elle ne spécifie pas suffisamment la différence entre existentielles et déclaratives. Mais c'est qu'elle est, en outre, inexacte, parce qu'elle ignore la possibilité réelle de combinaison ou alternance des cas génitif et accusatif marquant des objets du même type et dans des phrases semblables.

Tout d'abord, le cas génitif a des emplois différents de ceux qui sont liés à la négation, et tout à fait en accord avec son nom : il peut exprimer la partie d'un tout (au singulier et au pluriel), avec des substantifs, des indéfinis, des adverbes ou des numéraux. Et il apparait, dans d'autres situations, dans des expressions à sens impersonnel-passif. Mais surtout, pour ce qui nous intéresse, il apparait comme marque typique de l'objet de verbes transitifs sous l'effet de la négation. Par contre, cet emploi n'est pas exclusif du génitif, puisqu'il y a des cas de contraste avec l'accusatif. Et la même chose arrive dans des contextes affirmatifs, où le génitif (pur, sans contexte négatif) peut alterner avec l'accusatif (en manifestant les différences de signification qu'il existe entre He comprado el pan et He comprado pan).

2.2.2. Comme Grenoble (1992) le signale, l'alternance en contexte négatif (et en réalité cela concerne tous les cas d'alternance) dépend d'un certain nombre de circonstances, analysées au détail par Timberlake (1975). En effet, Timberlake propose de distinguer l'emploi de l'accusatif de celui du " génitif de négation » en faisant appel à la notion d' individuation (ce qui apparait aussi chez Hooper \& Thompson (1980), dans sa hiérarchie de la transitivité, où une plus grande individuation correspond à une plus grande transitivité). Pour Timberlake, le degré d'individuation du participant (le complément) et la probabilité d'apparition de l'accusatif sont directement proportionnels. Tout ceci, en rapport avec un ensemble de caractéristiques que je reproduis :

\begin{tabular}{|l|l|l|}
\hline (33) & Participant hierarchies & (individuation) \\
\hline & & +individuated/-individuated \\
\hline & properness: & proper/common \\
\hline
\end{tabular}




\begin{tabular}{|l|l|l|}
\hline & abstractness: & concrete/abstract \\
\hline & partitivity: & count/mass \\
\hline & animacy: & animate/inanimate \\
\hline & number: & singular/plural \\
\hline & definiteness: & definite/indefinite \\
\hline & negation: & neutral/emphatic negation \\
\hline \hline & focus: & topicalized/neutral \\
\hline & modification: & modified/unmodified \\
\hline
\end{tabular}

Les caractéristiques du prédicat sont aussi pertinentes :

\begin{tabular}{|l|l|l|}
\hline (34) & Event hierarchies & (scope or force of negation) \\
\hline & finiteness: & infinitive/finite \\
\hline & aspect: & imperfective/perfective \\
\hline & mood : & indicative/imperfective, \\
\hline & & conditional \\
\hline & status : & declarative/interrogative \\
\hline
\end{tabular}

61 Puisqu'il s'agit d'une propriété scalaire, une plus grande quantité de traits détermine une plus grande probabilité d'apparition d'un cas ou de l'autre.

62 Naturellement, l'essai de Timberlake est explicatif, ce qui fait que de son échelle d'individuation on ne peut pas déduire une norme d'assignation de cas, mais il a été reproduit ici à cause de l'importance des dénominations qui, d'un côté et de l'autre, rappellent la distinction générique entre défini et indéfini.

63 Ce qui m'intéresse le plus c'est de souligner que le génitif russe est, dans ses relations avec la négation (et malgré la dénomination " génitif de négation »), en concurrence avec le cas accusatif. Ils s'opposent pour une différentiation qui semble liée à ce qui, d'après Timberlake, pourrait être appelé l'individuation. Cette individuation n'est pas seulement liée à sa manifestation par une forme casuelle spécifique. Les faits d'ordre syntaxique sont également importants, en ce qu'ils constituent aussi des moyens de montrer les différents degrés d'individuation. Comme Timberlake, lui même, signale, un objet topicalisé, par exemple, placé en tête de phrase, a tendance à être plus "individué » du point de vue contextuel et syntaxique, ce qui, bien évidemment, rendra moins probable son association avec des cas qui se rapportent à la non-individuation (le génitif, dans ce cas). 
2.2.3. Dans l'étude, déjà classique, de Jakobson (1932) on trouve non seulement des interprétations relatives aux faits, mais aussi un ensemble d'exemples d'intérêt incontestable. Ainsi, quand Jakobson signale comme valeur principale de Nominatif la signification du sujet d'une action transitive, il présente des exemples où n'importe quel autre cas serait exclu pour exprimer une telle signification (35b). Tandis qu'il pourrait y avoir le cas génitif, comme dans (35a), en dehors de cette signification :

\begin{tabular}{|l|l|}
\hline (35) & a. nikogo [GEN] ne bylo (Il n'y avait personne) \\
\hline \hline & b. nikto [NOM] ne pel (Personne n'a chanté) \\
\hline & c. *nikogo ne pelo \\
\hline
\end{tabular}

Jakobson caractérisait les valeurs du génitif à partir d'une sorte de rôle central qui le vouait à signaler que la portée de la participation de l'objet était moindre que sa portée totale, sans que cela veuille dire dans son interprétation que la valeur " partitive » soit strictement fondamentale, comme son inclusion parmi les cas caractérisés par le trait de quantification pourrait nous le laisser croire. Des exemples de génitif sont classés suivant des emplois ou fonctions différenciés (je me bornerai à quelques situations de contraste de cas qui comportent des différences de signification):

A) Dans des phrases nominales, avec des exemples en général exclamatifs :

\begin{tabular}{|l|l|}
\hline (36) & vody, vody! [G]...no ja naprasno stradal'cu vodu [AC] povadal (Pouchkine \\
\hline & (De l'eau, de l'eau !... mais il tendait en vain l'eau au souffrant \\
\hline
\end{tabular}

B) Le génitif en position de sujet. Ce cas correspond, comme on voit dans (37), à ce qu'on aurait pu prévoir, d'après les conditions examinées quand on a étudié les contextes d'inaccusativité ou d'autres semblables. Avec une différence: l'ordre, en russe, semble être suffisamment remplacé par le système de marques de cas :

\begin{tabular}{|l|l|}
\hline (37) & a. ljudej [G] sobralos' (Il s'est réuni des gens) [V 3e sing., neutre] \\
\hline & $\begin{array}{l}\text { b. ljudi [NOM] sobralis' [la même chose, sans mettre en relief les gens] (les gens se sont } \\
\text { réunis) [V } 3^{\mathrm{e}} \text { pl., masc.] }\end{array}$ \\
\hline & c. sutok [G] bylo (Lermontof) (il y avait [beaucoup] de jeux) \\
\hline & d. sutki [N] byli [le nombre n'est pas spécifié] \\
\hline & e. nuzno spicek [G] [Il faut des allumettes) \\
\hline & f. nuzny spicki [N] [sans souligner son manque effectif] \\
\hline & g. otveta [G] ne prislo (Il ne vint pas de réponse) ${ }^{14}$ \\
\hline & h. otvet [N] ne prisel (La réponse ne vint pas) \\
\hline
\end{tabular}


C) Le « génitif de négation »:

\begin{tabular}{|l|l|}
\hline (38) & ne citaju gazety (Je ne lis pas de journal) \\
\hline (39) & ne nasel kvartiry (Il n'a pas trouvé de chambre) \\
\hline
\end{tabular}

L'apparition de ce génitif négatif, dans l'interprétation de Jakobson, semble être liée a une sorte d' "absence de l'objet» (que je me permets de réinterpréter comme manque de référentialité). Jakobson met ces faits en rapport avec des oppositions fondées, de façon caractéristique, sur le caractère défini ou indéfini de l'objet, c'est à dire, des oppositions entre Accusatif et Génitif, dans un contexte non négatif, et où la différence est établie entre les types d'objets suivant qu'ils sont préalablement connus dans le contexte linguistique ou la situation extralinguistique qui précèdent l'énoncé ou non :

\begin{tabular}{|l|l|}
\hline$(40)$ & a. prosit' deneg $[G]$ (pour demander de l'argent) \\
\hline \hline & b. prosit' den'gi $[\mathrm{A}]$ (pour demander l'argent) \\
\hline
\end{tabular}

D) À propos de la valeur « partitive », Jakobson signale que l'opposition Génitif-Accusatif présente un contraste net, qui ressemble entièrement à la distinction dont nous venons de parler :

\begin{tabular}{|l|l|}
\hline (41) & a. vypil vina [G] (Il a bu [un peu] de vin) \\
\hline & b. vypil vino [A] (Il a bu le vin $)^{15}$ \\
\hline
\end{tabular}

68 Comme on peut constater dans les exemples empruntés à Jakobson, l'aspect central qui semble différencier l'emploi de l'accusatif de celui du génitif (dans des situations d'alternance) est celui qui sépare les SN définis des indéfinis. Et cela arrive dans des contextes caractérisés par leur nature présentationnelle-existentielle, que l'on exprime la présence «nouvelle » des objets dont l'existence-référentialité n'était pas présupposée, ou qu'il s'agisse de références non spécifiques plus proches des lectures attributives que des référentielles.

Un fait curieux : aucun exemple ne présente de cas d'objets résultants ou effectués. Tous les cas de " génitif négatif » des exemples sont liés à des objets affectés, c'est à dire, ceux dont l'existence peut être supposée antérieure à l'action ou procès indiqué par le verbe. Naturellement, dans ces conditions, l'accusatif implique leur préexistence, tandis que le génitif ne l'implique pas, tout simplement ${ }^{16}$. Si les objets étaient des objets effectués ou résultants [construir una casa], l'action de la négation impliquerait le nonaccomplissement et donc la non-référentialité [no ha construído casa alguna]. Ou, si l'objet est contextuellement référentiel et préalablement connu, l'action de la négation impliquerait la non-réalisation de l'action [no ha construído la casa]. De son côté, la contrepartie affirmative impliquerait justement l'accomplissement, et pourrait très bien revêtir un caractère semblable aux constructions existentielles au cas où l'objet serait «nouveau» dans le contexte discursif-pragmatique [ha construído una casa]. Dans ce cas, 
l'indéfinitude est liée à la difficulté d'identification du référent, mais non pas à l'absence de référence ${ }^{17}$.

70 2.2.4. L'une des dernières études à propos de ce sujet, celle de Hentschel (1992), aborde les raisons du choix de cas et, en ce qui concerne la paire Génitif-Accusatif dans des objets de verbes niés, il fait remarquer que la conception habituelle qui associe le génitif à la nonréférentialité objective ou subjective du SN objet (à la manière de Jakobson ou Schaller (1978)), n'explique pas de façon satisfaisante les faits, et qu'une analyse à partir d'un corpus prouve que l'assignation de cas obéit à des raisons multifactorielles (que Hentschel n'explique pas assez, d'autre part). De ses remarques, on pourrait conclure que le génitif russe a une fonction [exclusive] de marque redondante de négation, et qu'il se trouve sur un chemin qui mène à sa disparition. Mais on ne trouve aucun argument réel contre l'association de l'accusatif avec la définitude, ni, en conséquence, du génitif avec l'indéfinitude. On pourrait même utiliser l'argumentation de Hentschel en faveur des critères de distinction référentielle entre les SN à l'Accusatif ou au Génitif, puisque seules des explications de ce genre pourraient rendre compte de quelque chose qui échappe à Hentschel : pourquoi cette marque redondante de négation apparaît dans certains cas et jamais dans d'autres? Ou une question encore plus simple : pourquoi une caractéristique que l'on suppose verbale, puisqu'elle se limite à la négation, apparait-elle réalisée dans la morphologie nominale de l'objet? Ce n'est pas la première fois que l'on confond la coapparition d'éléments avec la redondance ${ }^{18}$.

71 2.2.5. L'hypothèse la plus plausible, et aussi la plus simple, consiste à considérer que, dans les contextes qui nous intéressent, le génitif sert à marquer, dans une langue sans déterminants, un degré d'indéfinitude associé au SN. Il sert aussi à marquer que le SN se trouve sous la portée de la négation, indépendamment de sa position.

\subsection{Le partitif basque}

Dans le cas du basque, il existe aussi une association habituelle entre un cas, le partitif, et la négation.

2.3.1. Le partitif basque marque régulièrement les objets de verbes transitifs sous l'effet de la négation, dans les interrogatives et dans d'autres contextes généraux de nonspécificité. Étant donné que le basque est une langue du type ergatif qui possède un système structuré de détermination et aussi un système d'affixes casuels, les options théoriques pour marquer la non-spécificité ou l'indéfinitude pourraient être associées à n'importe lequel des deux systèmes. En fait, les fonctions semblent assez partagées, puisque le partitif entraîne la contrepartie négative des $\mathrm{SN}$ indéfinis, mais avec une modification en plus : la terminaison partitive n'est adjointe qu'au $\mathrm{N}$ dépourvu de toute marque de détermination. Ainsi, un SN peut présenter des degrés différents de détermination en ayant recours à l'affixation de l'article $[-a]$ ou au moyen de spécificateurs «définis» (bat, batzu,...), qui ont une signification semblable à celle des « indéfinis » de l'espagnol ou du français, par exemple.

Un syntagme au cas absolutif est, en réalité, un $\mathrm{SN}$ marqué seulement par la détermination et dépourvu d'affixe casuel explicite. N'importe quel autre cas (ergatif, sociatif, etc) entraîne l'adjonction de la forme affixale correspondant à un SN déjà préalablement déterminé. Par contre, le partitif (réalisé comme -ik), est obtenu, comme nous avons déjà dit, par l'adjonction de la forme affixale à la forme non déterminée du $\mathrm{N}^{19}$ . Voilà des exemples qui montrent des contextes différents d'apparition du partitif : 


\begin{tabular}{|l|l|}
\hline$(42)$ & Ez dut ogirik jango. (je ne mangerai pas de pain ${ }^{20}$ \\
\hline$(43)$ & Ogirik ba ahal dugu? (avons-nous du pain? \\
\hline$(44)$ & Badugu beste lanik. (Nous avons d'autres travaux \\
\hline$(45)$ & Gizonik ikusi duzu? (As-tu vu des hommes? \\
\hline
\end{tabular}
[izan] (mais avec une distribution qui n'est pas si régulière), les SN arguments de prédicats ne manifestent pas de cas morphologique différent pour les inaccusatifs et pour les objets de transitifs, étant donné que le comportement morphologique ergatif du basque implique en fait l'identification des deux cas comme non ergatifs. Si l'on examine quelques exemples avec un verbe tel que etorri (venir), qui s'associe à des sujets absolutifs et à l'auxiliaire izan, on peut distinguer les cas où le SN apparaitt sans article (adiskide) :

\begin{tabular}{|l|l|}
\hline$(47)$ & a. Zenbat adiskide etorri da (Combien d'amis est-il venu ?) \\
\hline & c. Bost adiskide etorri dira (Ils sont venus cinq amis) \\
\hline & d. Adiskiderik etorri al da ? (Est-il venu des amis ?) \\
\hline & e. Honera ez da adiskiderik etorri (Il n'est pas venu d'amis ici) \\
\hline
\end{tabular}

où l'on constate que le $\mathrm{N}$ adiskide doit être accompagné de spécificateurs tels que zenbat (combien) ou de numéraux (47b) pour pouvoir apparaitre sans la forme - $\boldsymbol{a}$ de l'article, alors que ceci est possible et nécessaire quand le contexte négatif (47e) ou interrogatif (47d) permet la forme du partitif, qui exclut la détermination et, en plus, ne s'associe qu'à la forme verbale de $3^{\text {ème }}$ pers. du singulier. 
80 adiskideak)

\begin{tabular}{|l|l|}
\hline$(48)$ & a. Ene adiskidea/adiskideak etorri da/dira (Mon/mes ami/s est/sont venu/s) \\
\hline & b. Etorri al da/dira ene adiskidea/k ? (Est-il venu mon ami ?/sont-ils venus mes amis ?) \\
\hline \hline & c. Ene adiskidea/k ez da/dira etorri (Mon ami n'est pas venu/ mes amis ...) \\
\hline
\end{tabular}

le partitif n'apparaît pas, et le verbe s'accorde au singulier et au pluriel suivant le nombre $\mathrm{du}$ SN sujet. La contrepartie négative de (48c) pourrait être

(48) c'. Ene adiskiderik ez da etorri (il n'est pas venu de mes amis)

avec une différence évidente : le SN ne peut pas être interprété comme référentiel défini et il s'accorde, bien entendu, au singulier.

81 Seuls les SN indéfinis sans détermination qui se trouvent sous la négation ou dans une interrogation sont marqués au partitif. En dehors de ces contextes, son apparition n'est même pas régie par les quantificateurs ${ }^{21}$, puisqu'ils agissent comme des modificateursdéterminants (antéposés ou postposés), ce qui produit des combinaisons syntagmatiques douées de détermination, même si le N n'a pas d'article. On peut constater, donc, que le partitif suppose l'existence d'un contexte adéquat et un SN indéfini/indéterminé.

Dans les phrases à verbe transitif, associé à des sujets ergatifs et des objets absolutifs (et avec l'auxiliaire ukan), on obtient des différences comme celle que l'on constate en (49), avec des absolutifs sans déterminant, et en (50), avec des SN au même cas mais déterminés :

\begin{tabular}{|l|l|}
\hline$(49)$ & a. Zenbat zakur eraman dute (Combien de chiens ont-ils emmené ?) \\
\hline & b. Zakur asko eraman dute (Ils ont emmené beaucoup de chiens) \\
\hline $\begin{array}{l}\text { c. Lau zakur eraman dituzte (dute) [dituzte pour l'objet pl. et dute sing.] (Ils ont emmené } \\
\text { quatre chiens) }\end{array}$ \\
\hline $\begin{array}{l}\text { d. Zakurrik eraman al dute? [dute parce qu'on ne peut pas accorder l'objet partitif au } \\
\text { pluriel] (Ont-ils emmené de chien ?) }\end{array}$ \\
\hline \begin{tabular}{l} 
e. Hemendik ez dute zakurrik eraman (Ils n'ont pas emmené de chien d'ici) \\
\hline$(50)$
\end{tabular} & $\begin{array}{l}\text { a. Aldameneko zakurra/k eraman dute/dituzte (Ils ont emmené le/s chien/s d'à côté) } \\
\text { côté ?) }\end{array}$ \\
\hline \hline & $\begin{array}{l}\text { d. Aldameneko zakurra/k ez dute/dituzte eraman (Ils n'ont pas emmené le/s chien/s d'à } \\
\text { côté) }\end{array}$ \\
\hline
\end{tabular}


83 partitif continue à apparaître de façon exclusive en absence de détermination, et il s'accorde au singulier avec le verbe (ce qui pourrait être interprété aussi comme un simple manque d'accord), tandis que les objets absolutifs, avec détermination, s'accordent au singulier et au pluriel.

Les pronoms indéfinis basques n'apparaissent pas marqués au partitif (bien qu'il existe des formes qui pourraient être considérées comme synthétiques, obtenues de la combinaison de la négation $(e z)$ et la forme indéfinie [ezer $=e z-z e r]$ :

2.3.3. Dans cette langue, le caractère indéfini et non spécifique d'un SN implique l'apparition d'un cas spécial partitif. D'après les exemples que nous avons examinés, la situation du basque confirme certains aspects de l'hypothèse inaccusative. D'une part, évidemment, parce que ses caractéristiques particulières faisaient déjà prévoir les ressemblances entre les objets de verbes transitifs et les sujets d'intransitifs : dans les deux cas les SN apparaissent marqués comme absolutifs, ce qui constitue la base même de la typologie ergative. Et pourtant l'intérêt des exemples ne s'arrête pas là, puisque nous avons constaté qu'un SN à interprétation indéfinie apparaît marqué comme partitif même si la structure où il se trouve et la fonction qu'il accomplit avaient fait prévoir l'absolutif (ce qui aurait été le cas si le SN avait eu une interprétation définie). Dans cette situation l'indéfinition entraîne aussi l'exclusion de la marque de détermination. Il s'agit là de SN indéfinis-indéterminés.

À mon avis, on peut en conclure que ce qui est important est l'association entre négation et cas partitif, et l'association entre EI et partitif, ou, ce qui revient au même, le rapport fondamental entre SN indéfinis et négation. Des exemples comme

(52) Ez dakit ogirik ote dagoen (Je ne sais pas s'il y a du pain)

87 où le SN partitif [ogirik] apparaitt comme argument d'une construction existentielle, ne sont possibles qu'en tant que cas où la négation affecte et marque un SN indéfini qui est aussi l'objet d'un verbe sous la portée de la négation [dakit]. La version affirmative de l'existentielle ne pourrait apparaître qu'avec un SN absolutif, comme en (53), contrairement au partitif de (54)

\begin{tabular}{|l|l|}
\hline$(53)$ & Badago ogia (Il y a du pain) \\
\hline \hline & Badago ogi bat (il y a un pain) \\
\hline$(54)$ & Ez dago ogirik (Il n'y a pas de pain) \\
\hline
\end{tabular}


2.3.4. Azkue ${ }^{22}$ disait que le partitif basque n'est employé qu'avec des patients et des accusatifs, ce qui était, assurément, sa façon à lui de dire que les SN marqués au partitif ne correspondaient jamais aux sujets des verbes actifs transitifs, mais seulement aux objets de ceux-ci et aux sujets des intransitifs, c'est à dire, à ce que la Grammaire Relationnelle appelle "Undergoers». Dans ce sens, le basque agit comme il était prévisible dans une langue ergative (au sens morphologique), et donc en accord avec l'hypothèse inaccusative: elle présente des uniformités entre les arguments d'inaccusatifs et les arguments objets de verbes actifs transitifs. Il y a cependant une régularité de plus, qui est liée à la négation et manifestée morphologiquement, si l'on rajoute la condition du caractère non défini et non déterminé des $\mathrm{SN}^{23}$.

\section{Conclusions}

En partant de l'observation du basque et du russe on peut conclure que, comme il avait été prévu, l'hypothèse inaccusative est essentiellement correcte, en ce qu'elle prévoit des comportements observables dans les langues: l'uniformité de comportement des SN arguments d'inaccusatifs et les SN objets de transitifs. Comme nous l'avions aussi prévu, l'EI a un plus grand relief dans son association avec la négation (avec des conséquences morphologiques) qu'avec l'inaccusativité.

3.1. Ce que l'on remarque dans les exemples que nous avons présentés est plus exactement une situation où le caractère défini où indéfini d'un SN n'est pas un effet mais la cause de son interprétation et des marques morphologiques qu'il reçoit. Et ce qui en réalité mérite une explication est la régularité avec laquelle les $\mathrm{SN}$ indéfinis reçoivent des marques spéciales quand ils sont sous la portée de la négation. Nous pouvons avancer, néanmoins, que si ce sont les $\mathrm{SN}$, à cause de leur traits, qui imposent leur conditions de fonctionnement syntaxique-morphologique, l'hypothèse de l'assignation inhérente de cas perd ce qui était peut-être son seul appui.

91 À mon avis, le caractère non défini d'un $\mathrm{SN}$ est une caractéristique liée à des phénomènes de référentialité qui entraîne des effets syntaxiques-morphologiques, et je n'accepte pas que ce soit le résultat de son rapport avec certains types de prédicats. Le cas qu'un SN de ce genre peut recevoir dépendra des langues, et d'après ce que nous avons vu, celles-là ont tendance à les différencier de façon spéciale quand ils se trouvent sous la portée de la négation ${ }^{24}$.

3.2. Par l'application d'une simple déduction, on peut parvenir à une série de déductionsconclusions, les unes liées aux langues que nous avons prises en exemple, et les autres, plus générales, qui surgissent de l'élargissement de l'interprétation à des langues de caractéristiques différentes (comme le français ou l'espagnol) :

1) Pour une langue du type ergatif, avec un système de marques casuelles et des déterminants comme le basque, l'hypothèse inaccusative coïncide en fait avec son comportement grammatical habituel. Son système de marques de cas signale la ressemblance des objets de verbes transitifs et des sujets de (certains) verbes intransitifs.

2) Il est très significatif, nonobstant, qu'avec une telle ressemblance déjà établie et formellement marquée, y apparaisse une marque de cas spéciale (le partitif) pour signaler, en plus, les interprétations non définies des SN qui se trouvent sous l'action de la négation et que, justement à cause de cela, ils n'ont pas de marque de détermination.

3) Pour une langue non ergative, avec un système de marques casuelles, mais sans 
déterminants, comme le russe, l'hypothèse inaccusative prévoit l'existence de certains cas de comportement semblable entre des arguments objets de verbes transitifs et des arguments de verbes inaccusatifs. Et pourtant il arrive que la véritable ressemblance de comportement a lieu entre des SN indéfinis, marqués par le cas génitif, et de façon caractéristique sous la négation. Par conséquent, la comparaison réelle doit être établie entre les SN indéfinis, puisque dans le cas où ils seraient définis l'opposition serait établie entre les cas nominatif et accusatif.

À partir de ces conclusions partielles, je crois que (étant d'accord avec les grandes lignes de l'hypothèse inaccusative, et précisément à partir de l'intuition de Belletti qui la met en rapport avec l'EI), il est permis de conclure qu'en réalité une telle hypothèse n'a de pouvoir explicatif que si les SN arguments comparés sont les indéfinis. Cependant, il a été prouvé aussi l'existence d'une tendance que l'on remarque dans les différentes langues, quand elles disposent de marques morphologiques casuelles, à marquer avec le même cas les SN qui, au delà de leur caractère indéfini, sans détermination, se trouvent sous l'effet de la négation.

Donc, la vraie ressemblance semble n'avoir lieu que dans les cas de SN indéfinis. Et cela est évident dans le fait que, morphologiquement, ils se présentent marqués de façon semblable dans des contextes et avec des formes particulièrement réservées aux SN indéfinis sous la portée de la négation. Il faudrait encore rajouter que tout ceci n'est pas exclusif des SN dans des contextes inaccusatifs, au sens élargi de Belletti, mais est valable pour d'autres types de contextes.

3.3. Si l'on réfléchit maintenant à des langues comme le français ou l'espagnol, qui pourraient être définies par leur manque de cas morphologique, mais qui ont des déterminants, l'ensemble des déductions précédentes nous pousserait à penser que, puisque ce ne sont pas des langues du type ergatif:

a) elles présenteront des réalisations de l'hypothèse inaccusative partielles, et bien entendu non identiques.

b) elles ne pourront pas marquer au moyen de cas les ressemblances que l'hypothèse prévoit. Elles seront donc obligées d'avoir recours à des procédés d'ordre de constituants et/ou des procédés qui impliquent leur système de déterminants.

c) elles peuvent, éventuellement, établir des marques spéciales pour certains cas. Et, à défaut de systèmes casuels, de telles marques devront utiliser justement les distinctions formelles de détermination qui sont à leur portée.

d) Puisque la fonctions de sujet, liée à la position préverbale, a tendance à exiger que les SN qui l'accomplissent soient définis, les SN indéfinis auront tendance à apparaître en position d'objets (c'est à dire, postverbale). Mais les stratégies à suivre changeront en fonction du paramètre du sujet nul.

3.4. Si nous retournons aux faits concrets, on conclut que les langues à sujet obligatoire ont tendance à utiliser des procédés qui ont comme résultat la conversion en non-sujets des SN indéfinis, en utilisant des constructions impersonnelles ou passivesimpersonnelles à sujet explétif.

Dans des langues à sujet nul les explétifs ne sont pas nécessaires, mais leur absence rend favorable l'établissement d'un accord sujet-verbe pour les SN indéfinis. Dans ce cas, on a recours à l'inversion de l'ordre du SN indéfini sujet.

Les constructions impersonnelles ou passives-impersonnelles produisent le même effet chez les deux types de langues : l'apparition postverbale du SN indéfini. Avec, en surplus, 
le fait que dans cette position il se trouve toujours sous l'éventuelle influence d'une négation verbale. Si cela arrive, les SN indéfinis postposés au verbe reçoivent une interprétation indéfinie qui pourrait être marquée comme non référentielle par recours au système de déterminants : la détermination zéro ${ }^{25}$.

Les constructions existentielles, de leur côté, présentent plus évidemment des exigences d'indéfinition chez les SN (comme nous avons vu précédemment). Il s'en suit que ce soient justement les constructions pour lesquelles les différentes langues ont choisi des formes verbales spécialisées, généralement associées à des expressions de type locatif et qui produisent des interprétations proches aussi de l'impersonnalité. Le résultat pour chacune d'entre elles, dépend du type de $\mathrm{V}$ choisi et du paramètre auquel la langue appartient ${ }^{26}$.

Dans le cas où une langue à sujet nul considérerait les $\mathrm{SN}$ indéfinis comme de vrais sujets (en établissant l'accord avec le verbe), on doit s'attendre a ce qu'elle ne puisse pas utiliser des marques prépositives ou qui auraient cette origine. Par contre, si la langue à sujet obligatoire considère les $\mathrm{SN}$ indéfinis comme des objets, elle pourrait développer une ou des marques spéciales d'indéfinition/non-référentialité : c'est le cas, à mon avis, de la forme de sans déterminant qu'on trouve en français (le de négatif, le de «litigieux » de Wilmet (1974)). La distribution de cette forme de (voir Gaatone (1992), ou Muller (1977)) correspond, dans des contextes négatifs, aux $\mathrm{SN}$ indéfinis non référentiel ${ }^{27}$ postposés au verbe (en fonction d'objet). Et cette correspondance a lieu, aussi bien quand la postposition est due à une structuration transitive que quand elle est due à n'importe quel procès d'inaccusativité, ou d'impersonnalisation, ou de structuration existentielle ${ }^{28}$.

Des exemples empruntés à Gaatone ((27)-(31)), on peut conclure que la forme de est devenue une vraie marque de SN indéfini objet atteint par l'effet de la négation. C'est à dire, elle est devenue un marqueur de portée de la négation ${ }^{29}$.

Et si l'on accepte cette dernière interprétation, une donnée nouvelle confirmerait la tendance dans des langues très diverses à établir des mécanismes morphologiques qui marquent de la même façon les $\mathrm{SN}$ qui se trouvent sous l'effet de la négation. Une tendance qui confirme aussi que l'hypothèse inaccusative (si elle veut maintenir sa généralité et sa capacité de prédiction) doit être restreinte aux SN indéfinis. De la même façon, cette tendance soutient l'hypothèse selon laquelle le caractère défini ou indéfini n'est pas un effet obtenu à cause des propriétés de certains prédicats, mais plutôt la cause de certaines structurations marquées du point de vu syntaxique.

3.5. De leur côté, les données morphologiques des langues de différents types ont tendance à confirmer que les marques spécifiques, des cas ou de la détermination, s'associent normalement, non aux SN indéfinis sans plus, mais de façon spéciale, aux SN indéfinis qui se trouvent sous la portée de la négation.

L'analyse des phénomènes relatifs à la négation permet donc de conclure que ce sont les contextes en rapport avec elle qui rendent favorable l'apparition de comportements et marques spéciales pour indiquer le caractère non spécifique, indéfini ou non référentiel d'un SN, et ceci indépendamment du type de prédicat qui le gouverne. La notion de " partitif» de Belletti, plutôt que comme cas inhérent, mérite d'être récupérée comme mise en relief de la situation spéciale d'un SN (sans préjuger le cas qu'il reçoit) dont le caractére indéfini (et d'avantage s'il est interprété comme non référentiel) donnent lieu à des restrictions évidentes par rapport aux possibilités, plus grandes, des SN définis. 


\section{BIBLIOGRAPHIE}

ANSCOMBRE, J.-Cl. (1986) : «L'article zéro en français : un imparfait du substantif ? », Langue Française $72: 4-39$.

ANSCOMBRE, J.-Cl. (1986) : «Article zéro, termes de masse et représentation d'événements en français contemporain », Recherches Linguistiques XI : 5-34.

ANSCOMBRE, J.-Cl. (1991) : «L'article zéro sous préposition », Langue Française 91 : 24-39.

ANSCOMBRE, J.-Cl. (1991) : « La détermination zéro : quelques propriétés », Langages 202 : 103-124.

ATTAL, P. (1976) : «A propos de l'indéfini “des" : Problèmes de représentation sémantique ", Le Français Moderne, $44:$ 126-142.

BABBY, L.H. (1978): Negation and Subject Case Selection in Existential Sentences. Indiana Linguistics Club. Bloomington, Indiana.

BARWISE, J. y COOPER, R. (1981): « Generalized quantifiers and natural language », Linguistics \& Philosophy 4: 159-219.

BELLETTI, A. (1988): « The Case of Unaccusatives », Linguistic Inquiry 19: 1-34.

BELLETTI, A. (1990): Generalized Verb Movement. Aspects of Verb Syntax. Torino, Rosemberg \& Sellier.

BELLETTI, A. et RIZZI, L. (1982): « On the syntax of ne. Some theoretical implications », The Linguistic Review 1: 117-155.

BOSQUE, I. (1987) : « Clases de sujetos tácitos », Philologica. Homenaje a Antonio Llorente, Universidad de Salamanca, 1989, vol. 2, pp. 91-111.

BOSQUE, I. (1980) : Sobre la negación. Madrid, Cátedra.

BURZIO, L. (1981): Intransitive verbs and italian auxiliaries. Thèse Doct. du MIT, Cambridge, Mass.

BURZIO, L. (1986): Italian Syntax. A Government and Binding Approach. Dordrecht, Reidel.

COMRIE, B. (1978): « Genitive-accusative in Slavic: The rules and their motivation », International Review of Slavic Linguistics 3: 27-43.

GAATONE, D. (1970) : « La transformation impersonnelle en français », Le Français Moderne 38 : 389-341.

GAATONE, D. (1992) : « De négatif entre la syntaxe et la sémantique. Réflexions sur quelques propriétés du déterminant de », Langue française 94 : 93-102.

GALMICHE, M. (1986) : « Note sur les noms de masse et le partitif », Langue Française 72 : 40-53.

GIVON, T. (1992): « The grammar of referential coherence as mental processing of instructions ", Linguistics 30: 5-55.

GRENOBLE, L.A. (1992): « Double negation in Russian », Linguistics 30: 731-752.

GUÉRON, J. (1991) : « Le clitique SE et la grammaire des pronoms indéfinis », dans Guéron, J. et J.Y. Pollock (éds.) Grammaire générative et syntaxe comparée. Paris, Editions du CNRS : 191-213. 
HAWKINS, J.A. (1978) : Definiteness and Indefiniteness. London, Croom Helm.

HELDNER, C. (1981) : La portée de la négation. Stockholm, Norstedts.

HELDNER, C. (1992) : « Sur la quantification négative », Langue Française 94 : 80-92.

HENTSCHEL, G. (1992) : « Zum Ein_uß der Konstituentenfolge auf die Kasuswahl im Russischen », Lingua 87 : 231-255.

HERNANDEZ PARICIO, F. (1985) : Aspectos de la negación. León, Univ. de León.

HIGGINBOTHAM, J. (1983): « Logical Form, Binding, and Nominals », Linguistic Inquiry 14: 395-420.

HIGGINBOTHAM, J. (1985): « On Semantics », Linguistic Inquiry 16: 547-594.

HIGGINBOTHAM, J. (1987): "Indefiniteness and Predication », dans Reuland, E.J. et A.G.B. ter Meulen (eds.) The Representation of (In)definiteness. MIT Press, Cambridge, Massachussets.

HOOPER, P.J. et S.A. THOMPSON (1980): « Transitivity in grammar and discourse », Language 56:251-299.

JACKENDOFF, R. (1990): Semantic Structures. MIT Press, Cambridge, Mass.

JAKOBSON, R. (1936) : « Beitrag zur allgemeinen Kasuslehre : Gesambedeutungen der russischen Kasus ", Travaux du Cercle Linguistique de Prague 6 : 240-288.

KEENAN, E. (1984): « Semantic correlates of the ergative/absolutive distinction », Linguistics 22:197-223.

KEENAN, E. et J. STAVI (1986): « A semantic characterization of natural language determiners », Linguistic \& Philosophy 9/3: 253-326.

LEGENDRE, G. (1988): « Two classes of unergatives in French? », Proceedings of the 24th Meeting of the Chicago Linguistic Society.

LEGENDRE, G. (1989): « Unaccusativity in French », Lingua 79: 95-164.

MAY, R. (1985): Logical Form. Its Structure and Derivation. The MIT Press, Cambridge, Mass.

MILSARK, G. (1974): Existential Sentences in English. D. Diss., MIT, Cambridge, Mass.

MILSARK, G. (1977): « Towards an explanation of certain peculiarities of the existential construction in English », Linguistic Analysis 3: 1-29.

MULLER, Cl. (1977) : « A propos de de partitif », Lingvisticae Investigationes I/1 : 167-198.

MULLER, Cl. (1991) : La négation en français. Genève, Droz.

OLIE, A. (1984) : «L'hypothèse de l'inaccusatif en français », Lingvisticae Investigationes 8 : 363-401. ORTIZ DE URBINA, J. (1990): Some Parameters in the Grammar of Basque. Dordrecht, Foris.

PERLMUTTER, D.M. (1978): «Impersonal passives and the unaccusative hypothesis », Proceedings of the Fourth Annual Meeting of the Berkeley Linguistics Society, 157-189.

PICABIA, L. (1986) : « Remarques sur l'interprétation indéfinie », Recherches Linguistiques XI : 227-245.

RIGAU, G. (1988) : « Els predicats no verbals i l'efecte d'inespecifitat », Estudi General 8 : 51-64.

RUWET, N. (1988) : « Les verbes météorologiques et l'hypothèse inaccusative » dans C.B. Benveniste, A. Chervel et M. Gross (eds.), Grammaire et histoire de la grammaire. Mélanges a la mémoire de Jean Stéfanini. Aix-en-Provence. Publications de l'Univ. de Provence, 383-402.

RUWET, N. (1990) : « Des expressions météorologiques », Le français Moderne LVIII : 43-97. 
SIEWIERSKA, A. (1991): Functional Grammar. London, Routledge.

SERBAT, G. (1981) : Cas et fonctions. Paris, P.U.F.

SCHALLER, H.W. (1978) : Das direkte Objekt in verneinten Sätzen des Russischen. Frankfurt, Lang.

SZABOLCSI, A. (1984): «From the Definiteness Effect to Lexical Integrity », Proceedings of the Groningen Round Table on the Definiteness Effect.

TASMOWSKI-DE RYCK, L. (1990) : « ...En semble... ou ...semble en... », Le français moderne 58 : 28-42.

TIMBERLAKE, A. (1975): « Hierarchies and the genitive of negation », Slavic and East European Journal 19: 123-138.

VENDLER, Z. (1967): Linguistics and Philosophy. New York, Cornell University Press.

WIERZBICKA, A. (1988): The Semantics of Grammar. Amsterdam, J. Benjamins.

WILMET, M. (1974) : «Sur de inverseur », Travaux de Linguistique et de Littérature XII/1 : 301-323.

WILMET, M.(1986) : La détermination nominale. Paris, P.U.F.

ZRIBI-HERTZ, A. (1987) : « La réflexivité ergative en français moderne », Le Français Moderne 55 : 23-54.

\section{NOTES}

1. Dans le domaine du français, il y a des travaux spécifiquement consacrés à prouver l'existence de phénomènes liés à l'inaccusativité (Olié (1984), Zribi-Hertz (1987) Legendre (1988, 1989), Ruwet (1988, 1990), Tasmowski (1990), etc.). Ils n'avaient pas, cependant, d'intérêt pour le sujet étudié ici.

2. La terminologie est relativement compliquée et obscure. D'habitude on trouve Definiteness Effect, dans le sens de Milsark et Belletti: dans ce cas, il est question de l'exigence d'une interprétation indéfinie du SN. Dans d'autres cas (Rigau (1988)), nous trouvons «Effet d'inespécificité », vu comme l'exigence d'une interprétation spécifique. Guéron (1991:192) parle de «effet indéfini », qui doit être interprété comme la condition qui « exclut toute phrase sans sujet argumental dans laquelle le complément d'objet est interprété comme spécifique » (ce qui n'empêche qu'elle analyse de façon assez étrange les exemples espagnols, puisqu'elle présente comme exemple d'absence d'effet indéfini l'espagnol se comió las manzanas, dans le sens impersonnel, mais elle oublie que cette interprétation est beaucoup plus fréquente avec des objets indéfinis: Se comió tarta, Se habló de política, etc. (il faut dire aussi que l'interprétation impersonnelle de son exemple est loin d'être la plus naturelle).

Nous maintiendrons ici, par sa simplicité, la dénomination de EI de Guéron, dans le sens de Milsark-Belletti. Il faut noter, dès maintenant, que nous croyons qu'il faudrait séparer les situations où il y a une « exigence » d'EI de celles où il n'y en a que la « possibilité ».

3. Milsark souligne la possibilité d'apparition de ce qu'il appelle "weak determiners » ( $a$, some, many), et l'impossibilité des «strong determiners", qu'il considère quantificationnels. Ce qui permet l'apparition des premiers dans une construction existentielle est leurs interprétations non quantificationnelles. Les types de détermination établis par Milsark sont de la même nature (et terminologie) que ceux de Barwise et Cooper (1981), ou ceux de Keenan et Stavi (1986), qui parlent d'indéfinis et définis.

4. Rigau (1988) signale qu'en catalan il est plus facile de trouver des exemples de ce genre, ce qui fait qu'elle parle d'un "effet d'inespécificité» qui, inversement à ce qu'il a été vu, exige l'apparition de SN obligatoirement déterminés. Quant au EI, on peut trouver des phrases comme 
celles de (11) et (12) acceptables en catalan :

i) a. Hi ha un noi

b. Hi ha el noi

c. Hi ha en Pere

Et, d'autre part, des exemples où c'est le caractère référentiel ou spécifique qui est exigé :

ii) a. El capellà no hi és

b. *Un capellà hi és

c. ${ }^{*}$ Cap capellà no hi és

Comme on peut l'imaginer, la vraie différence concerne l'interprétation existentielle (avec EI) ou d'autres interprétations. Ainsi, (12d) est acceptable en catalan parce qu'il ne s'agit pas d'une phrase existentielle, qui doit être traduite à l'espagnol, non pas avec le verbe haber impersonnel, mais avec estar.

5. L'italien possède aussi des cas à valeur double. À côté d'exemples comme (12e), on peut trouver des exemples comme :

i) a. C'è l'uomo del pane

b. C'è l'uomo vecchio

Comme dans le cas du catalan, ces verbes sont accordés aux syntagmes qui suivent ( $\mathrm{C} i$ sono degli uomini in ascensore).

6. Belletti cite l'explication «conceptuelle» de Szabolcsi (1984). Elle admet, en passant, la possibilité, évidente, que l'EI puisse concerner aussi les verbes transitifs.

7. Ce serait un exemple de ce que Bosque (1987) appelle des «impersonnelles accordées». Des contrastes semblables : Son las tres, Il est trois heures...

8. La dénomination à été tirée, de même que l'interprétation, d'une extension de ce que Belletti observe dans le cas du partitif finnois.

9. Ce qui prédit un type de lecture existentielle des dits SN indéfinis, indépendamment du nombre morphologique qu'ils puissent manifester (s'ils en manifestent). Dans la même ligne d'argumentation on aurait pu penser aux avantages de considérer que les SN définis présentent un type de quantification proche de la quantification universelle (qui ne serait évident qu'avec le pluriel). Hernández (1985) proposait que les quantificateurs négatifs sont des universels constitués à partir d'existentiels.

10. Qui ou qu'est ce qui sélectionne la lecture de ce SN affecté ? Le seul sélecteur possible est la construction impersonnelle, comme un tout. Si cela est vrai, les langues qui font accorder les SN au verbe (et qui produisent moins probablement des impersonnelles) devront manifester des comportements divergents. En espagnol, par exemple, il y a des passives toujours accordées comme :

i) Fue asesinado un hombre / Juan.

Fue encontrado petróleo / el perro.

Tandis que les constructions impersonnelles reflètent que le SN est marqué comme accusatif

ii) Se ha asesinado $a$ un hombre.

L'EI n'est pas obligatoire, donc. À ce propos, il faut dire que dans le travail de Belletti il n'est pas toujours clair si on a affaire au Cas abstrait ou au cas morphologique. Elle établit cette différence dans l'exemple allemand Es gibt einem man, où elle justifie l'accusatif du SN postverbal comme réalisation de surface du partitif inhérent. D'autre part, Belletti admet l'existence d'autres contextes où des verbes transitifs pourraient être des potentiels assignateurs de cas partitif ( L'altro giorno ho mangiato spaghetti /Spaghetti, l'altro giorno ne ho mangiato), ouvrant définitivement la porte à la possibilité d'admettre que l'EI des existentielles pourrait ne pas être l'indéfinitude des SN dans d'autres contextes. Belletti (1990) étudie le ASC (Absolute Small Clauses) de l'italien et suppose que les SN postposés aux participes passés de verbes inaccusatifs reçoivent leur cas nominatif du V, puisque dans ces constructions on ne trouve pas d'EI. 
11. Siewierska (1991:168) analyse l'inaccusativité en rapport avec l'établissement de «new topics» (NewTop).

12. La situation de ces $\mathrm{SN}$, que Gaatone considère «l'objet direct d'un verbe complexe (auxiliaire + infinitif), parfois analysé comme sujet sous-jacent de l'infinitif" (p. 102), est, évidemment, différente. Il serait intéressant de les mettre en rapport avec les thèses qui soutiennent que les «small clauses" (les petites phrases) n'admettent pas de sujet indéfini.

13. Il s'agit d'un aspect bien connu et fréquemment étudié. Parmi d'autres, on peut consulter les travaux de Jakobson (1936), Timberlake (1975), Schaller (1978), Comrie (1978), Wierzbicka (1988) et Hentschel (1992).

14. C'est à Serbat (1981:116) qu'on a emprunté les traductions de (37g y h). Ses commentaires à propos du schéma des cas russes de Jakobson ne sont pas pertinents ici, puisque nous sommes plus intéressés par les données que par les interprétations.

15. Jakobson signale, cependant, que les êtres animés ne peuvent apparaître que très rarement au génitif singulier (otvedal kuricy [il a goûté de la poule]), ce qui fait que l'opposition AccusatifGenitif pour les êtres animés disparaît dans la plupart des paradigmes, sous la forme syncrétique de Génitif. La distribution la plus habituelle serait :

i) a. kupil kartiny $[\mathrm{A}]$ (il a acheté des tableaux)

b. kupil kartin [G] (il a acheté [une série de] tableaux)

ii) kupil losadej [A-G] (il a acheté des chevaux)

16. Comme on peut voir, l'implication de la non-existence de l'objet (c'est à dire de son référent) est le résultat de la combinaison de $\mathrm{N}$ au génitif avec la négation. Le génitif tout seul peut être marqueur d'un certain degré de référence ou de définitude, mais nous ne pouvons pas lui attribuer des caractéristiques qui dépendent de l'effet de la négation, étant donné que le génitif russe possède d'autres fonctions qui n'impliquent pas de marques supplémentaires.

17. Une autre distinction dont il faut tenir compte est celle qui sépare les verbes qu'impliquent ce que Gaatone (1992) appelle «l'existence du référent de l'objet", ce qui sans doute pourra conditionner les comportements. Ou des distinctions comme celles de Vendler (1967), qui expliquent les différences existantes entre l'interprétation comme activité :

i) Juan ha comido chocolate.

et l'accomplissement

ii) Juan se ha comido el chocolate.

Cette dernière interprétation (reflétée par l'espagnol) interdit la présence d'objets directs indéfinis :

iii) *Juan se ha comido chocolate.

18. Dans le contexte de notre travail, on peut signaler que dans des phrases existentielles du type il y $a$, le russe n'utilise le génitif que sous la négation (avec la forme niet sans verbe, s'il s'agit du présent, ou avec la négation simple et des formes impersonnelles du verbe pour le passé et le futur). Il y a aussi des cas de libre choix entre expression personnelle et impersonnelle (et si la construction est négative, le génitif doit apparaître dans ce dernier cas, mais le nominatif pour les formes accordées). Devant une telle situation, il semble que les constructions russes seraient plutôt apparentées à des distinctions semblables en espagnol (Había médicos y enfermeras / Estaban los médicos y las enfermeras, et les négations No había medicos ni enfermeras / No estaban los médicos ni las enfermeras).

19. La plupart des exemples sont tirés d'Alvarez Enparantza, J.L. [Txillardegui] : Euskal gramatika. San Sebatián, Ediciones Vascas, 1978.

20. La traduction des exemples du basque sera toujours littérale à fin de souligner l'accord verbal.

21. Sauf dans des combinaisons avec asko et gutxi /gitxi : eskerrik asko (merci beaucoup) azurrik asko ta maminik gitxi (beaucoup d'os et peu de chair).

22. Azcue, R.M. de : Diccionario vasco-español-francés. Bilbao, 1905 (imprimé à Tours). 
23. À partir du modèle de GB, Ortiz de Urbina (1990) présente une systématisation qui n'est pas trop éloignée de la nôtre. D'après lui, tous les verbes basques morphologiquement intransitifs qui sélectionnent l'auxiliaire izan (être) (car il y en a d'autres qui sélectionnent ukan (avoir)) ont comme arguments des objets profonds, qui reçoivent le cas absolutif (de même que les objets des transitifs) ou partitif (sous la négation, et aussi comme les objets des transitifs). Les problèmes d'assignation de cas restent toujours sans solution, à mon avis, dans le travail d'Ortiz, puisqu'il faut toujours trouver le moyen d'assigner deux cas (absolutif et partitif) à des SN qui sont des sujets ou des objets dans le cas des intransitifs, mais toujours des objets dans le cas des transitifs.

24. Dans de telles circonstances, l'hypothèse de l'assignation de cas inhérent pourrait devenir une sorte d'artifice établi comme système d'identification de SN en position d'objet. Mais pour cela il existe d'autres alternatives (qui ont recours à des systèmes d'indexation) d'analyse des faits de quantification et portée liés à la Forme Logique (Voir, par exemple, Higginbotham (1983, 1985 y 1987)).

25. Détermination zéro liée à des distinctions de nombre plus complexes :

i) a. Por aquí no pasan camiones [comptable]

b. Por aquí no pasa camión alguno

ii) a. Aquí no venden café [masse]

b. *Aquí no venden café alguno [acceptable seulement s'il s'agit de différentes sortes de café]

Et conditionné par des phénomènes caractéristiques du système de détermination de chaque langue :

iii) a. Yo no leo el periódico [indéfini-générique]

b. Yo no leo periódicos

c. Yo no leo periódico *(que me guste)

26. Par exemple, si le prédicat est du type qu'on pourrait dénommer ESSERE, les SN associés auront tendance à être considérés comme des sujets syntaxiques (accordés). Par contre, s'il s'agit de prédicats du type HABERE, les SN auront tendance à être considérés comme de vrais objets (avec des marques ou des caractéristiques d'accusatifs, dans des langues non ergatives bien entendu). L'influence de la diachronie sur ce point est claire.

27. Bien qu'il y ait une évidente absence de précision dans mon travail à propos des termes «défini» et «référentiel», il faut tenir compte de ce que «non-référentiel» peut être interprété comme «qui n'a pas de référent» ou comme «qui n'a pas de lecture référentielle» (en usage attributif, par exemple), si ce n'est pas la même chose quand on parle de SN. Les idées de Givon (1992), qui sont reproduction de celles qu'il a déjà présentées maintes fois, correspondent assez bien à notre concept de référence.

28. Autrement dit, les $\mathrm{SN}$ indéfinis qui en français sont précédés de la forme de plus une forme du déterminant doivent être nettement différenciés du de «négatif». Les combinaisons de+det ont une distribution différente et, évidemment, elles ne supposent pas la non référentialité du SN. Seul le de négatif (sans déterminant, où avec déterminant zéro) marque le nom positivement comme non référentiel. Cette capacité provient sans doute de son association avec la négation, mais elle permettrait de le considérer comme un autre membre du système de la détermination. Il resterait en tout cas un doute : les SN introduits par de négatif, sont-ils de vrais SN ? Si oui, ils sont défectifs, comme ils le sont du point de vue fonctionnel (ils n'ont de clitique correspondant que en), comme étaient défectifs les partitifs basques (qui ne s'accordent pas au verbe). Évidemment, le manque de lecture référentielle sert d'obstacle à leur interprétation comme de vrais arguments.

Sur les problèmes de la détermination zéro, on peut consulter avec profit le travail réalisé par Anscombre, parmi d'autres.

29. On peut noter la différente portée dans le contraste simple :

i) a. Je n'ai pas reçu le livre hier soir

b. Je n'ai pas reçu de livre hier soir 
Comme Gaatone (1992 :98), «si l'on admet l'existence d'un lien nécessaire entre de N et NÉG, toute non-occurrence de de en dépit de la présence de NÉG doit signifier qu'il n'y a pas association entre $\mathrm{N}$ et NÉG, autrement dit que ce nom ne se trouve pas dans le champ de la négation.»

\section{RÉSUMÉS}

Ce travail a pour but principal de mettre en rapport l'étude de la négation et celle des faits regroupés sous le nom d'hypothèse inaccusative, en prenant comme point de départ la version de l'hypothèse présentée par Belleti (1988). L'observation du basque et du russe nous a permis de conclure que l'hypothèse inaccusative est essentiellement correcte, en ce qu'elle prévoit des comportements observables dans les langues : l'uniformité de comportement des SN arguments d'inaccusatifs et les SN objets de transitifs. Mais on constate également que l'Effet Indéfini associé par Belleti à un cas inhérent partitif - a un plus grand relief dans son association avec la négation (avec des conséquences morphologiques : le partitif dans le cas du basque et le génitif dans le cas du russe) qu'avec l'inaccusativité. L'analyse des phénomènes relatifs à la négation permet de conclure que ce sont les contextes en rapport avec elle qui rendent favorable l'apparition de comportements et marques spéciales pour indiquer le caractère non spécifique, indéfini ou non référentiel d'un SN, et ceci indépendamment du type de prédicat qui le gouverne.

The main aim of this paper is to relate facts concerning negation and those which regard the Inaccusative Hypothesis, as stated by Belleti (1988). In observing Russian and Basque Languages, we can conclude that the Inaccusative Hypothesis is largely correct in that it predicts that NP "arguments" of Inaccusative Verbs behave like the NP objects of Transitive Verbs. But it is also possible to observe that the Indefinite Effect appears more strongly when it is linked with negation than with Inaccusative Verbs and has morphological consequences: the Partitive Case in Basque and the Genitive in Russian. Having analysed the phenomena related to negation, we can conclude that negative contexts favour particular sorts of behaviour and particular markers, indicating the non specific, indefinite or non referential character of an NP, whichever predicate is governing it.

\section{AUTEUR}

\section{FRANCISCO HERNÁNDEZ-PARICIO}

Departamento de Lingüística General e Hispánica, Universidad de Zaragoza 